\title{
Implications of Different Feedback Types on Error Perception and Psychological Reactance
}

\author{
Patrick Ehrenbrink \\ Quality and Usability Lab \\ Technische Universität Berlin \\ 10587 Berlin, Germany \\ patrick.ehrenbrink@tu- \\ berlin.de
}

\author{
Xin Guang Gong \\ Quality and Usability Lab \\ Technische Universität Berlin \\ 10587 Berlin, Germany \\ xinguanggong@icloud.de
}

\author{
Sebastian Möller \\ Quality and Usability Lab \\ Technische Universität Berlin \\ 10587 Berlin, Germany \\ sebastian.moeller@tu-berlin.de
}

\begin{abstract}
In Human Computer Interaction, appropriate feedback is especially important when an error occurs. This work compares implications for user's psychological reactance and acceptance of two different types of error and their representations through feedback. Reactance and acceptance levels were measured after presenting feedback that allows a conclusion on the cause of an occurred error and feedback that fails to do so. It was found that reactance levels were significantly higher and acceptance levels significantly lower in the latter case. Suggestions for appropriate system design are discussed.
\end{abstract}

\section{Author Keywords}

Psychological Reactance; Acceptance; Errors; System Feedback

\section{ACM Classification Keywords}

H5.2. Information interfaces and presentation (e.g., HCI): User Interfaces.

\section{INTRODUCTION}

System feedback can have important implications for HCI. Especially in connection with system- or user errors, adequate feedback can provide valuable hints for the user to resolve or adequately handle an undesired system response. Usually, guidelines on feedback claim that it should be complete and provided in a way that the user fully understands outcomes (Hartson et al., 2012). Unfortunately, such recommendations are often not followed, but error codes or very generic messages are displayed, instead. Besides the obvious negative aspects of such a practice, e.g. no or weak situation awareness of the user, we argue that also psychological consequences can follow. One of such consequences can be that users become reactant. Psychological reactance is a construct from social psychology. It was first introduced by (Brehm, 1966). Reactance is described as an affective state that can occur if a person perceives a loss of freedom of choice or control (Brehm, 1966). It is likely to

This work is licensed under a Creative Commons Attribution-NonCommercial International 4.0 License.

Copyright is held by the owner/author(s).

OzCHI '16, November 29 - December 02, 2016, Launceston, TAS, Australia

ACM 978-1-4503-4618-4/16/11.

http://dx.doi.org/10.1145/3010915.3010994. be manifested in a mixture of anger and negative cognitions (Dillard et al., 2005) towards the stimulus. Additionally, the level of reactance that a person experiences is also due to his or her proneness to become reactant (Brehm et al., 1981). Therefore, proneness to reactance can also be regarded as a trait reactance. A given system that induces reactance in its users is likely to suffer decreased acceptance, as was observed for example in public health campaigns (Dillard et al., 2005). Similar effects could be shown for the context of online recommendation systems (Lee et al., 2009). Furthermore, it was found that social agency of persuasive systems can increase reactance (Roubroeks et al., 2011). Apart from this, the concept of reactance has not received much attention as a directly measured variable in the context of HCI, so far. There is however some work that mentions the concept but did not directly measure reactance (Laschke et al. 2014; Stieger et al. 2007).

The way in which system design, and in particular system feedback design, influences user reactance is not yet known.

A study that investigates the implications of two different types of errors and according feedback on user judgment about a smart TV is presented here. The different error types and their representation through feedback are as follows. The first error type represents an interpretation error of a speech command. This means that the user will be able to see that the speech command was recognized successful, but interpreted wrongly. The wrong interpretation then leads to a wrong execution of the command. Nevertheless, the execution is consistent with the false interpretation. Through the feedback that is given, users can get an explanation about why the smart TV did not perform the task that the user intended.

The second type of error was implemented in such a way that the user does not get a sufficient explanation on why the error might have occurred. Through system feedback, the user can see that the speech command was recognized correctly and the interpretation was correct, as well. However, the smart TV still performs a false execution of the command. We assume that this will lead to a state of confusion and a feeling of lost control over the situation. According to reactance theory (Brehm et al., 1981), this would then result in a higher state of psychological reactance and also in a decrease of acceptance of the smart TV. Three hypotheses are stated for the current study: 


\section{Hypothesis 1}

A sufficient number of errors during interaction decreases the users' control over the smart TV. This will be perceived by the users and then induce a heightened state of reactance in both error conditions, compared to a baseline condition that does not contain any errors.

\section{Hypothesis 2}

Even though users suffer a certain loss of control over the smart TV in the condition with the first error type, system feedback still provides a rational explanation on how the error occurred (via a false interpretation of the command). In the condition with the second error type, no such explanation is provided. It is assumed that the lack of sufficient explanation of the error leads to a state of confusion and a perception of lessened control over the situation in the users. This could then be observed in a higher state of users' reactance in the condition with the second error type, compared to both other conditions.

\section{Hypothesis 3}

Former studies hinted that reactance has a negative influence on acceptance towards the stimulus (Dillard et al., 2005). Acceptability (Moeller et al., 2007), which is the potential to induce acceptance of the smart TV will be accessed alongside state reactance. If reactance can have a negative influence on acceptance, then acceptability of the smart TV should be lowest in the condition with error type two.

Should the hypotheses be confirmed and errors can cause reactance, this will provide some implications for developers. Reactance is usually an undesired effect for any type of communication. If reactance can be shown to be influenced by the type of error handling and decrease acceptability, developers should commit sufficient attention to error handling to keep reactance levels of users are kept as low as possible.

\section{METHODS}

\section{Participants}

36 adults that were recruited via a participant database took part in the experiment. All of them were between 18 and 44 years old $(M=27.44$ years, $S D=5.60)$. Gender was balanced and resulted in a total of 18 females and 18 males. For the statistical analysis, the dataset of one participant was dropped because it was an outlier. This resulted in a total number of 17 participants for group I (baseline and first error type) and 18 participants for group II (baseline and second error type).

\section{Procedure}

The experiment was a partial between- partial withinsubjects design. All participants interacted with the prototype of a smart TV that used a smartphone as a remote control. Users could pose commands in natural language to the smartphone via a push-to-talk button. The commands were then processed and executed by the smart TV. Interaction consisted of a set of 17 tasks per condition that were stated in a way that the participants had to come up with the proper formulation of the commands by themselves. An example of a task is: "Search for action movies from the 80s." Participants were allowed to try to solve each task twice. During the interaction, the smart TV showed three types of feedback. A) The user command as interpreted by the natural language understanding software, e.g. "display the program for tomorrow", B) the interpretation of the command e.g. "program for tomorrow" and C) the actual execution of the command e.g. a list showing the program for tomorrow. A screenshot of the interface, including the three feedback types, is shown in Figure 1. At the beginning of the experiment, the instructor explained all three feedback types and their meaning to the participants, using an example query and oral explanations.

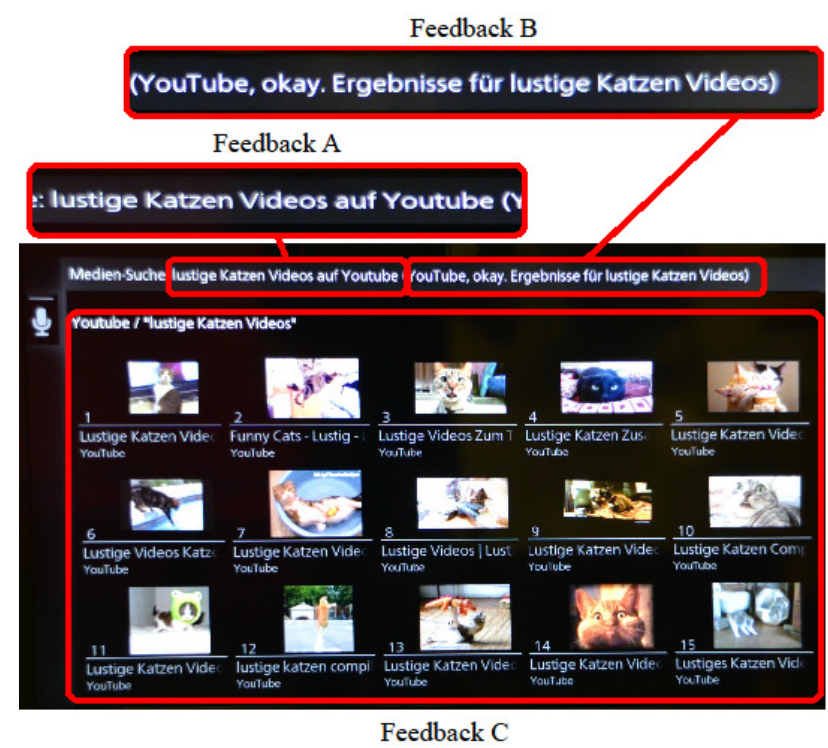

Figure 1. Interface of the Smart TV that was used. Feedback A shows the original speech command as recognized by the speech to text input. Feedback $B$ shows the interpretation of the seech command. Feedback $C$ shows the execution of the command in question.

The experiment had three conditions. The first condition (C1) was the baseline, where feedback was not manipulated and the smart TV acted as intended. In the second condition (C2), feedback A remained untouched, whereas feedback B was manipulated to commit the first error type (interpretation error). Feedback $\mathrm{C}$ was following the (false) interpretation that was shown in feedback B. In this condition, the error in feedback B can serve as an explanation for the false execution that is obvious from feedback C. In the third condition (C3), feedback A remained untouched, as well. Also, feedback B showed a correct interpretation of feedback A. Only feedback $\mathrm{C}$ showed a false content that was executed. This condition was designed to represent a dissonance between the interpretation of the user command and the execution of the smart TV, thereby not providing an explanation on why the error might have occurred. The error frequency of $\mathrm{C} 2$ and $\mathrm{C} 3$ was scripted to be $50 \%$, while no error would occur in the first two and last two trials of each condition. All participants were assigned to one of two groups (I and II) at random. Members of group I interacted with the smart TV in condition $\mathrm{C} 1$ and $\mathrm{C} 2$, whereas members of group II interacted with the smart TV in condition $\mathrm{C} 1$ and $\mathrm{C} 3$. The order of the two conditions within each group was changed for every 
participant in order to avoid any sequence effects. Also, all participants passed a training phase and thereby learned the appropriate commands that were to be used. Only a few errors that were not artificially introduced occurred during the experiment. After the interaction, all participants completed a set of questionnaires. Among those questionnaires was the P.851 (Moeller et al., 2007) that was used to provide a judgment of acceptability and includes items like "I would use the system again in the future" and a questionnaire for measuring state reactance. The reactance questionnaire is not yet published. See Table 1 for the included items.

\begin{tabular}{|c|c|}
\hline No. & Item (English Translation) \\
\hline 1 & The [System] is simply bad! \\
\hline 2 & $\begin{array}{l}\text { I would like to never see or hear anything of that } \\
\text { [System] again. }\end{array}$ \\
\hline 3 & I don't even want that [System] as a present. \\
\hline 4 & $\begin{array}{l}\text { The whole concept of the [System] is a poor } \\
\text { approach. }\end{array}$ \\
\hline 5 & The [System] frustrates me. \\
\hline 6 & The [System] makes me angry. \\
\hline 7 & $\begin{array}{l}\text { I get mad when I have to interact with the } \\
\text { [System]. }\end{array}$ \\
\hline 8 & $\begin{array}{l}\text { When I only see that [System], I burst with } \\
\text { anger. }\end{array}$ \\
\hline
\end{tabular}

Table 1. English translation of the items that were used to assess reactance. A previous study showed that the questionnaire has a high internal consistency (Cronbachs $\alpha$ of 0.90 for items 1-4:"Negative Cognitions" and 0.95 for items 5-8:"Anger").

\section{RESULTS}

The dimension acceptability of P.851 and the reactance scores of the first measure between the two groups I and II were analysed with a Mann-Whitney U test because Levene's Test for Equality of Variances was significant. The Mann-Whitney $U$ Test indicated that acceptability was not significantly different in $\mathrm{C} 1$ of the two groups I and II. Also, the Mann-Whitney U Test indicated that the level of reactance, as well as its two factors "negative cognitions" and "anger" were not significantly different in the first measures of the two groups I and II. Paired Samples T-Tests were performed to analyse differences of reactance and its factors between the first and the second measurement of the groups I and II. There was a significant difference in the scores for the first reactance measure and the second reactance measure of group I. There was also a significant difference in the scores for the first reactance measure and the second reactance measure of group II. The reactance scores of the second measurement of the two groups I and II were analysed with a Mann-Whitney U test, because Levene's Test for Equality of Variances was significant. The MannWhitney U Test indicated that the level of reactance in group II was greater than the level of reactance in group I. A summary of the measures in reactance and acceptability can be seen in Table 2. Furthermore, descriptive statistics are provided in Table 3.

\begin{tabular}{|c|c|c|c|}
\hline Conditions & Factor & $t / U$ & $\mathbf{P}$ \\
\hline \multirow{4}{*}{$\mathrm{C} 1$ (I)-C1(II) } & N. Cognitions & $\mathrm{U}=122.5$ & 0.309 \\
\hline & Anger & $\mathrm{U}=137$ & 0.589 \\
\hline & Reactance & $\mathrm{U}=128.5$ & 0.415 \\
\hline & Acceptability & $\mathrm{U}=140.5$ & 0.678 \\
\hline \multirow{4}{*}{$\mathrm{C} 1(\mathrm{I})-\mathrm{C} 2(\mathrm{I})$} & N. Cognitions & $t(16)=-2.18$ & 0.045 \\
\hline & Anger & $\mathrm{t}(16)=-3.59$ & 0.002 \\
\hline & Reactance & $\mathrm{t}(16)=-3.12$ & 0.007 \\
\hline & Acceptability & $t(16)=4.51$ & 0.000 \\
\hline \multirow{4}{*}{$\begin{array}{l}\text { C1(II)- } \\
\text { C3(II) }\end{array}$} & N. Cognitions & $\mathrm{t}(17)=-2.54$ & 0.021 \\
\hline & Anger & $\mathrm{t}(17)=-3.05$ & 0.007 \\
\hline & Reactance & $\mathrm{t}(17)=-2.95$ & 0.009 \\
\hline & Acceptability & $\mathrm{t}(17)=3.73$ & 0.002 \\
\hline \multirow{4}{*}{ C2(I)-C3(II) } & N. Cognitions & $\mathrm{t}(33)=-2.54$ & 0.016 \\
\hline & Anger & $\mathrm{t}(33)=-2.20$ & 0.015 \\
\hline & Reactance & $\mathrm{U}=81.5$ & 0.018 \\
\hline & Acceptability & $t(33)=2.24$ & 0.032 \\
\hline
\end{tabular}

Table 2. Overview of the results for the tests of significance. For cases in which a Paired Sample T-Test was performed, $t$ is reported. For cases in which a Mann-Whitney $U$ Test was performed, $U$ is reported. Group number is written in brackets.

The Pearson Correlation of reactance with acceptability was calculated for both groups independently. An overview of the accessed correlations can be seen in Table 4.

\begin{tabular}{|l|c|c|}
\hline & Reactance (I) & Reactance (II) \\
\hline Acceptability (I) & $-0.49 *$ & n.a. \\
\hline Acceptability (II) & n.a. & $-0.73 * *$ \\
\hline
\end{tabular}

Table 4. Overview of the correlations between Reactance (R.) and Acceptability (Acc.). Groups are written in brackets. Only correlations of variables that were accessed within subjects were analyzed. Significant correlations (below 0.05 ) are marked with a star, highly significant correlations (below 0.001) are marked with two stars.

\section{DISCUSSION}

Results show significant differences in reactance and its components anger and negative cognitions between all three conditions. Also, it was tested if there is a significant difference between the two groups I and II in condition $\mathrm{C} 1$. No such differences could be observed. Therefore it can be concluded that there is no observable difference between the two groups that could serve as an explanation of the differences between the conditions $\mathrm{C} 2$ 


\begin{tabular}{|l|l|l|l|l|l|l|l|l|l|}
\hline \multirow{2}{*}{ Condition } & \multirow{2}{*}{$\mathbf{N}$} & N. Cognitions & \multicolumn{2}{|c|}{ Anger } & \multicolumn{2}{c|}{ Reactance } & \multicolumn{2}{|c|}{ Acceptability } \\
\cline { 4 - 10 } & & Mean & SD & Mean & SD & Mean & SD & Mean & SD \\
\hline C1(I) & 17 & 1.62 & 0.52 & 1.57 & 0.58 & 1.60 & 0.44 & 3.74 & 0.54 \\
\hline C1(II) & 18 & 1.99 & 0.91 & 1.78 & 0.82 & 1.88 & 0.80 & 3.42 & 0.79 \\
\hline C1(I\&II) & 35 & 1.81 & 0.76 & 1.68 & 0.71 & 1.74 & 0.66 & 3.58 & 0.69 \\
\hline C2 & 17 & 1.90 & 0.63 & 1.93 & 0.62 & 1.91 & 0.51 & 3.22 & 0.55 \\
\hline C3 & 18 & 2.64 & 1.04 & 2.56 & 1.02 & 2.50 & 0.99 & 2.70 & 0.78 \\
\hline
\end{tabular}

Table 3. Descriptive statistics of Negative Cognitions, Anger, Reactance and Acceptability in the three conditions. Group number is written brackets.

and C3. Acceptability was measured for the proposed effect of reactance on acceptance. Results support the claim, that a system which induces reactance can thereby suffer a decrease of acceptability.

\section{Hypothesis 1}

The first hypothesis can be confirmed. It could be proven, that the two conditions that included errors induced significantly more reactance compared to the condition that did not include artificially added errors. This is true for reactance and both of its factors anger and negative cognitions.

\section{Hypothesis 2}

The second hypothesis can be confirmed, as well. In C2, in which an explanation for the error was provided via Feedback B, significantly lower levels of reactance were observed, compared to the condition in which feedback B did not provide an adequate explanation for the error. A likely explanation that is consistent with reactance theory is that users got an impression of less control over the situation because they could not explain the error via the given feedback. However, no additional measurements are available to proof or disproof this.

\section{Hypothesis 3}

The third hypothesis can be confirmed. There is a significant decrease in acceptability of the smart TV between the first measurement and the second measurement for groups I and II. Also, acceptability of the smart TV is significantly lower for the condition with error type two, compared to the condition with error type one. This is consistent with the levels of reactance that were measured in all conditions. The condition with error type two induced the highest levels of reactance, while the acceptability rating was lowest. For the condition without artificially induced errors, the observed levels of reactance are lowest, while the acceptability rating of the smart TV was highest.

Developers should always provide sufficient information if an error occurs, so that users are able to infer on the error-source. This will likely prevent or lessen the amount of users' reactance if an error occurs. The results also underline the importance of the psychological construct reactance as a metric for HCI in general. Reactance is rarely accessed in HCI research, even though several studies pointed at its relevance for the field (Roubroeks et al., 2011; Lee et al., 2009; Stieger et al., 2007). In the current study, reactance was moderated by the design of the error message. However, the design of messages of other sorts, such as persuasive attempts (Dillard et al., 2005; Lee et al., 2009) or even the human likeness of the messenger agent (Roubroeks et al., 2011) have been known to influence reactance, as well. Our analysis has shown significant correlation between reactance and acceptability. A strong negative correlation that was highly significant was observed in group II with error type two. Also, a significant negative correlation was observed in the group with error type one. Overall, the observed correlations support the claim that reactance has a negative influence on acceptance. Acceptability is not equal to acceptance but it closely resembles the concept of potential acceptance of a given system on a selfreporting level. The findings imply that strategies that reduce reactance can also be used to increase acceptance of devices, not only in the context of error messages. Such strategies include adjustment of social agency (Roubroeks et al. 2011) and optionality (Stieger et al. 2007). After finding the effect of differences in errorfeedback on reactance in this scenario, we are continuing our research on reactance and its implications for system design. We are currently studying the influence of trait reactance on users' design preferences of different system parameters. Those parameters include human-likeness, directness of language in messages and the level of detail in which information is presented. We hope that this will lead to a collection of guidelines for better addressing users' personality traits in adaptive systems.

\section{CONCLUSIONS}

We were able to show significant increases in state reactance between the untouched condition and the condition that contained errors. Also, it was shown that the type of error message can have a significant influence on the amount of reactance and acceptance. We conclude that system design that aims at minimizing reactance will also positively influence acceptance. Therefore, developers should try to emphasize users' control.

\section{ACKNOWLEDGMENTS}

This work was supported by BMWi, grant no. 01MG13001G, Universal Home Control Interfaces@ConnectedUsability (UHCI) and by BMBF, Software Campus, grand no. 01IS12056, Sozialpsychologische Aspekte von Smart Homes (SPASH). 


\section{REFERENCES}

Brehm, J. W. A theory of psychological reactance. Academic Press (1966).

Brehm, S. S., and Brehm, J. W. Psychological reactance: A theory of freedom and control. Academic Press (1981).

Dillard, J.P., and Shen, L. On the nature of reactance and its role in persuasive health communication. Communication Monographs 72(2) (2005), 144-168.

Hartson, R., and Pyla, P. S. The UX Book: Process and guidelines for ensuring a quality user experience. Elsevier (2012).

Laschke, M., Diefenbach, S., Schneider, T., \& Hassenzahl, M. (2014, October). Keymoment: Initiating behavior change through friendly friction. In Proceedings of the 8th Nordic Conference on HumanComputer Interaction: Fun, Fast, Foundational (pp. 853-858). ACM.
Lee, G., and Lee, W. J. Psychological reactance to online recommendation services. Information \& Management 46(8) (2009), 448-452.

Moeller, S., Smeele, P., Boland, H. and Krebber, J. Evaluating spoken dialogue systems according to defacto standards: A case study. Computer Speech and Language 21 (2007), 26-53.

Roubroeks, M., Ham, J., and Midden, C. When artificial social agents try to persuade people: The role of social agency on the occurrence of psychological reactance. International Journal of Social Robotics 3(2) (2011), 155-165.

Stieger, S., Reips, U.-D., and Voracek, M. Forcedresponse in online surveys: Bias from reactance and an increase in sex-specific dropout. Journal of the American society for information science and technology 58(11) (2007), 1653-1660. 